\title{
The relationships among perceived stress, conflict resolution styles, spousal support and marital satisfaction during the COVID-19 quarantine
}

\author{
Rabiye Akın Işık ${ }^{1} \cdot$ Yunus Kaya $^{2}$ \\ Accepted: 17 January 2022 / Published online: 9 February 2022 \\ (c) The Author(s), under exclusive licence to Springer Science+Business Media, LLC, part of Springer Nature 2022
}

\begin{abstract}
This study investigated the relationships among perceived stress, conflict resolution styles, spousal support and marital satisfaction of heterosexual married couples during the 2019 Novel Coronavirus (COVID-19) quarantine. This was a descriptive study that employed a correlational survey model. The sample consisted of 511 Turkish married couples recruited using snowball sampling. Data were collected online using a demographic characteristic questionnaire, the Perceived Stress Scale, the Conflict Resolution Styles Scale, the Spousal Support Scale, and the Marital Life Scale. The mean age of participants was $37.00(\mathrm{SD}=7.88)$ years. The mean duration of marriage of participants was $11.15(\mathrm{SD}=8.97)$ years. Higher marital satisfaction was associated with lower perceived stress, lower negative conflict resolution style, higher spousal support, and higher positive resolution styles. The Conflict Resolution Styles Scale and Spousal Support Scale scores explained 48.3\% of the total variance of the Marital Life Scale, indicating that higher positive conflict resolution styles and higher spousal support were significantly associated with higher marital satisfaction. The COVID-19 quarantine has resulted in changes in marital life and family dynamics. Stress, negative conflict resolution style, and a lack of spousal support during the COVID19 quarantine contribute to marital dissatisfaction.
\end{abstract}

Keywords COVID-19 $\cdot$ Perceived stress $\cdot$ Conflict resolution $\cdot$ Spousal support $\cdot$ Marital satisfaction $\cdot$ Heterosexual married couples

\section{Introduction}

The novel Coronavirus disease (COVID-19) broke out in Wuhan, the capital of Central China's Hubei province, at the end of 2019 and has taken hold of the entire world since then (Xiang et al., 2020). The virus is transmitted between people through the exchange of respiratory droplets when in close contact with each other, causing pneumonia, acute respiratory syndrome, and death, particularly among elderly

Rabiye Akın Işık

akinrabia35@gmail.com

Yunus Kaya

yuunus.kaya@gmail.com

1 Department of Obstetrics and Gynecelogic Nursing, Faculty of Nursing, Hacettepe University, Sıhhıe Campüs, Ankara 06100, Turkey

2 Faculty of Health Sciences, Aksaray University, Child Development Department, Aksaray, Turkey persons with chronic diseases (CDC, 2020; Wu \& McGoogan, 2020). The COVID-19 disease has become a global health problem and has infected millions of people and caused tens of thousands of deaths. Therefore, the World Health Organization (WHO) has classified it as a pandemic (WHO, 2020), and governments of countries worldwide have taken several measures to prevent its spread (ShakespeareFinch et al., 2020; Zhu et al., 2020). Turkey announced its first confirmed case of COVID-19 on March 11, 2020, and has taken several preventive measures to curb the spread of the virus. Among these measures are school closure, the transition to distance education, flexibility in working hours (public and private), work shifted to the online space and working from home, and lockdowns (institutions and workplaces). In order to prevent contagion, stay-at-home orders were given for people 20 years old and younger and those over 65 years, and curfews for all age groups in certain large cities on weekends were also applied (Karataş, 2020).

These measures have drastically changed the daily life of people everywhere. People remained in quarantine, either 
by their personal decisions or due to the decisions of their governments. The COVID-19 pandemic has affected people physically, mentally, and socially (Armstrong et al., 2020; Shakespeare-Finch et al., 2020; Zhu et al., 2020). The fear of becoming infected, the increase in the number of positive cases and deaths, social isolation, substantial uncertainties regarding the nature of the disease, and the dynamics of the pandemic have caused stress, anxiety, and psychosomatic conditions in people. Further, the increased uncertainty surrounding the virus, rapid course of the disease, and having loved ones infected or killed by the virus have exacerbated already-existing mental problems. Voluntary or mandatory quarantine deprived people of the social support that would have otherwise helped them cope with stress and anxiety, thereby increasing the severity of depression, anxiety, obsessive-compulsive disorders, and other psychosomatic conditions (Liu et al., 2020; Wang et al., 2020; Zhu et al., 2020).

The fear of becoming infected, mental strains, stress, anxiety, social isolation, changes in activities of daily living, and financial problems caused by the COVID-19 pandemic have affected domestic and marital life, often leading to family conflicts, domestic violence, and marital dissatisfaction (Gulati \& Kelly, 2020; Huang \& Zhao, 2020). The closure of workplaces, layoffs, and the ongoing stay-at-home orders resulting from the COVID-19 pandemic have led to numerous difficulties and disruption in the gender-based roles of married couples at home. Over $89 \%$ of Turkish citizens report being Muslim and 74\% of them are Sunni. In contemporary Turkish society, females are more concerned with domestic responsibilities and taking care of children, while males take on the responsibility of supporting the household financially. The changes in daily life that have arisen due to the pandemic have affected these responsibilities and gender roles. In many cases, this situation has led to an increase in the stress levels of spouses and their expectations of support from one another (Akbaş \& Dursun, 2020; Ünal \& Gülseren, 2020). Spouses meet each other's physical, mental, and social needs, such as sex, love, respect, value, and support. However, the COVID-19 pandemic has caused stress, anxiety, fear, and social isolation, thereby resulting in marital and domestic problems (Gulati \& Kelly, 2020; Karataş, 2020). Spouses who were able to cope with the challenges of the pandemic likely built a stronger bond and began trusting each other more (Prime et al., 2020). However, those who were unable to cope with the challenges of not only the pandemic but also the challenges associated with a strained marriage (Abbas et al., 2019; Soylu \& Kağnıc1, 2015), thereby exacerbating their mental problems and resulting in domestic violence and divorce. Therefore, this study investigated the relationships among perceived stress, conflict resolution styles, spousal support, and marital satisfaction of married couples during the COVID-19 lockdown. The study proposes the following hypotheses:
Hypothesis 1: The mean scores of the Perceived Stress Scale, Conflict Resolution Styles Scale, Spousal Support Scale, and Marital Life Scale vary according to the demographic characteristics of the participants (such as age, sex, education level, income level, etc.).

Hypothesis 2: Higher marital satisfaction is correlated with both lower perceived stress and negative conflict resolution styles.

Hypothesis 3: Higher marital satisfaction is correlated with both higher positive conflict resolution styles and spousal support.

Hypothesis 4: Perceived stress and negative conflict resolution styles negatively predict marital satisfaction.

Hypothesis 5: Positive conflict resolution styles and perceived spousal support positively predict marital satisfaction.

\section{Method}

\section{Design}

This descriptive study employed a correlational survey model to determine the relationship among perceived stress, conflict resolution styles, spousal support, and marital satisfaction of heterosexual married couples during the COVID19 lockdown period.

\section{Participants}

The study population was comprised of married couples who quarantined together during the COVID-19 pandemic in Turkey. The sample consisted of 511 Turkish married couples who were recruited using snowball sampling, a nonprobability sampling method. For snowball sampling, advertisements were used to recruit couples from social media (e-mail, WhatsApp, Instagram, or Facebook). The inclusion criteria were (1) voluntary participation and (2) being married for at least six months. The exclusion criteria were (1) living separately during the COVID-19 lockdown, (2) refusing participation, and (3) being married for less than six months. Eleven participants were excluded from the study because the duration of their marriage was less than six months and they did not live together during the COVID-19 lockdown period.

\section{Procedure}

The present study was approved by the Aksaray University Human Research Ethics Committee. Permission was obtained from the Ministry of Health of the Republic of Turkey. The study data were collected between July 1, 2020 and July 30, 2020 using an online questionnaire survey 
due to nationwide preventive measures and restrictions. The scales were prepared on Google Forms, and an online questionnaire link was sent (over e-mail, WhatsApp, Facebook, or Instagram) to all participants. The initial participants were asked to send the link to other married couples they knew (snowball sampling). Prior to participation, all couples were informed of the purpose and procedure of the study and online and written consent was obtained from those who agreed to participate. The data were exported from Google Forms to Google Sheets and then to an Excel sheet. Participants took approximately $30 \mathrm{~min}$ to complete the questionnaires.

\section{Instruments}

The data were collected online using a demographic characteristic questionnaire, the Perceived Stress Scale (PSS), the Conflict Resolution Styles Scale (CRSS), the Spousal Support Scale (SSS), and the Marital Life Scale (MLS).

\section{Demographic Characteristics Questionnaire}

The demographic characteristics questionnaire developed by the researchers consisted of 11 items including age, sex, education level, income level, duration of marriage, anxiety level, and perceived challenges during the COVID-19 pandemic.

\section{The Perceived Stress Scale}

The Perceived Stress Scale (PSS) developed by Cohen et al., (1983) is a self-reported measure of perceived stress, feelings, and thoughts in the past month. The PSS was adapted to Turkish by Eskin et al. (2013). The PSS consists of 14 items scored on a 5-point Likert-type scale (" $1=$ Never" to " $5=$ Very Often"). The total score ranges from 14 to 70 , with higher scores indicating higher stress levels. The PSS has a Cronbach's alpha of 0.84 in its original form, the Cronbach's alpha and McDonald's Omega values were both found to be 0.86 in this study.

\section{The Conflict Resolution Styles Scale}

The Conflict Resolution Styles Scale (CRSS) developed by Özen (2006) is a measure of the conflict resolution styles adopted by married couples. The CRSS comprises 25 items scored on a 6-point Likert-type scale (" $1=$ Strongly disagree" to " $6=$ Strongly agree"). The scale has four subscales: the Positive Conflict Resolution Style (PCRS), Negative Conflict Resolution Style (NCRS), Subordination Conflict Resolution Style (SCRS), and (4) Retreat Conflict Resolution Style (RCRS). The subscale scores are taken into account for assessment. A higher score on a subscale indicates more common conflict resolution styles that correspond to that subscale (Özen, 2006). The CRSS subscales have a Cronbach's alpha of $0.75-0.81$ in its original form, Cronbach's alpha and McDonalds' Omega values ranged from 0.70 to 0.80 in this study.

\section{The Spousal Support Scale}

The Spousal Support Scale (SSS) was developed by Yildirım (2004) to assess the level of perceived spousal support. The SSS consists of 27 items scored on a 3-point Likert-type scale (" $1=$ Does not describe me at all" to " $3=$ Describes me well"). The SSS has four subscales: (1) emotional support, (2) instrumental and informational support, (3) appraisal support, and (4) social support. The total score ranges from 27 to 81 , with higher scores indicating higher perceived spousal support. The SSS has a Cronbach's alpha of 0.95 in its original form, the Cronbach's alpha and McDonalds' Omega values were found to be 0.96 in this study.

\section{The Marital Life Scale}

The Marital Life Scale (MLS) was developed by Tezer (1994) to assess how satisfied couples are with their marriage. The MLS comprises 10 items scored on a 5-point Likert-type scale (" $1=$ Strongly disagree," to " $5=$ Strongly Agree"). The total score ranges from 10 to 50, with higher scores indicating higher marital satisfaction (Tezer, 1994). The MLS has a Cronbach's alpha of 0.91 in its original form, the value of Cronbach's alpha for this study was 0.70 , while McDonalds' Omega value was found to be 0.75 in this study.

\section{Ethical Considerations}

The study was approved by the Aksaray University Human Research Ethics Committee (Decision No/Date: 2020.06-29/22.06.2020). Permission was obtained from the Ministry of Health of the Republic of Turkey (2020-0605T10_22_33). The procedures used in this study adhere to the tenets of the Declaration of Helsinki.

\section{Data Analysis}

Data were analyzed using the SPSS $₫ 23.0$ (IBM Corporation, Armonk NY, USA) for Windows ${ }^{\circledR}$ at a significance level of 0.05 . The numbers and percentages were used for descriptive analysis. The Kolmogorov-Smirnov test was used for normality testing. An independent sample t-test and a one-way analysis of variance (ANOVA) were used to determine between-group differences by demographic characteristics. Further, Tukey's test was used for pairwise group comparisons to determine the source of the differences. In addition, Spearman's correlation coefficient was 
used to determine the relationship among variables. Multiple regression analysis was used to determine the effect of conflict resolution styles and spousal support on marital satisfaction.

\section{Results}

The mean age of participants was $37.00(\mathrm{SD}=7.88)$ years $(\min =22 ; \max =70)$. Among the participants, $75.54 \%$ were females, $79.84 \%$ had a bachelor's degree or higher, and $50.88 \%$ had a moderate income $(48,000-72,000$ Turkish Liras). The mean duration of the marriage of participants was $11.15(\mathrm{SD}=8.97)$ years $(\min =1 ; \max =50)$. Further, $78.66 \%$ of the participants were living in cities with curfew ordinances, $83.17 \%$ had children $(61.88 \%$ had more than two), and $63.79 \%$ were not employed during the COVID-19 pandemic. Participants had a mean anxiety score of 7.10 $(\mathrm{SD}=2.45)(\min =1 ; \max =10)$ at the onset of the COVID19 pandemic and $6.00(\mathrm{SD}=2.53)(\min =1, \max =10)$ in the last week. The mean score of anxiety about health was $6.40(\mathrm{SD}=2.62)(\min =1, \max =10)$. For participants, the challenges of the COVID-19 pandemic were emotional and mental stress $(69.66 \% ; \mathrm{n}=356)$, having to postpone important plans $(52.44 \% ; \mathrm{n}=268)$, financial loss or job loss $(20.35 \%, \mathrm{n}=104)$, marriage and family problems $(12.13 \%$, $\mathrm{n}=62$ ), and having themselves tested positive or having a loved one tested positive for or died from COVID-19 $(7.82 \%, \mathrm{n}=40)$. Twelve participants $(2.34 \%)$ reported having no problems, and ten participants (1.95\%) took up new hobbies and spent quality time with their families during the lockdown.

Further, sex, place of residence, having children, and income level affected participants' mean PSS score in Table $1(p<0.05)$. Female participants had a higher mean PSS score than males $(t=-2.84, p=0.004)$. Participants living in cities with curfew ordinances had a higher mean PSS score than those who were not $(t=2.72, p=0.007)$. Participants without children had a higher mean PSS score than those with children $(t=-2.02, p=0.04)$. Low-income participants had a higher mean PSS score than those with high income $(F=8.19, p<0.0001)$. Age, sex, education level, having children, and income affected participants' mean SSS score in Table $1(p<0.05)$. Younger participants had

Table 1 Perceived stress, spousol support and marital life levels according to participants demographic characteristics

\begin{tabular}{|c|c|c|c|c|c|c|c|}
\hline $\begin{array}{l}\text { Demographic } \\
\text { characteristics }\end{array}$ & & PSS M \pm S.D & Analysis Results & SSS M \pm S.D & Analysis Results & MLS M \pm S.D & Analysis Results \\
\hline Age & $\begin{array}{l}<30^{\mathrm{a}} \\
30-40^{\mathrm{b}} \\
40-50^{\mathrm{c}} \\
\geq 50^{\mathrm{d}}\end{array}$ & $\begin{array}{l}42.03 \pm 5.51 \\
40.57 \pm 7.26 \\
39.65 \pm 7.17 \\
39.22 \pm 6.83\end{array}$ & $\begin{array}{l}F=2.43 \\
p=0.06 \\
\eta^{2}=0.01\end{array}$ & $\begin{array}{l}71.21 \pm 11.17 \\
68.23 \pm 12.28 \\
63.35 \pm 13.39 \\
64.39 \pm 13.93\end{array}$ & $\begin{array}{l}F=7.91 \\
p<0.0001 \\
\mathrm{a}>\mathrm{c}, \mathrm{a}>\mathrm{d} \\
\mathrm{b}>\mathrm{c}, \mathrm{b}>\mathrm{d}^{*} \\
\eta^{2}=0.05\end{array}$ & $\begin{array}{l}34.80 \pm 3.97 \\
33.15 \pm 4.97 \\
31.90 \pm 4.70 \\
32.92 \pm 4.44\end{array}$ & $\begin{array}{l}F=6.36 \\
p<0.0001 \\
a>c, a>d^{*} \\
\eta^{2}=0.04\end{array}$ \\
\hline Sex & $\begin{array}{l}\text { Male } \\
\text { Female }\end{array}$ & $\begin{array}{l}38.93 \pm 7.54 \\
41.06 \pm 6.71\end{array}$ & $\begin{array}{l}t=-2.87 \\
p=0.004\end{array}$ & $\begin{array}{l}71.39 \pm 10.20 \\
65.98 \pm 13.28\end{array}$ & $\begin{array}{l}t=4.77 \\
p<0.0001\end{array}$ & $\begin{array}{l}34.66 \pm 4.59 \\
32.61 \pm 4.75\end{array}$ & $\begin{array}{l}t=4.23 \\
p<0.0001\end{array}$ \\
\hline Education & Primary $^{\mathrm{a}}$ & $40.07 \pm 7.02$ & $F=0.85$ & $67.09 \pm 15.11$ & $F=4.21$ & $33.61 \pm 4.62$ & $F=2.25$ \\
\hline Level & $\begin{array}{l}\text { Secondary } \\
\text { High }^{\mathrm{c}} \\
\text { Bachelor }^{\mathrm{d}} \\
\text { Post-Graduate }^{\mathrm{e}}\end{array}$ & $\begin{array}{l}42.31 \pm 5.63 \\
40.94 \pm 7.90 \\
40.01 \pm 6.80 \\
41.27 \pm 7.10\end{array}$ & $\begin{array}{l}p=0.51 \\
\eta^{2}=0.01\end{array}$ & $\begin{array}{l}56.13 \pm 15.88 \\
63.85 \pm 12.39 \\
68.48 \pm 12.54 \\
67.85 \pm 11.56\end{array}$ & $\begin{array}{l}p=0.001 \\
a>b, a>c \\
d>b, d>c \\
e>b, e>c^{*} \\
\prod 2=0.04\end{array}$ & $\begin{array}{l}29.44 \pm 5.30 \\
32.61 \pm 5.26 \\
33.33 \pm 4.75 \\
33.25 \pm 4.33\end{array}$ & $\begin{array}{l}p=0.048 \\
a>b, c>b, \\
d>b, e>b^{*} \\
\eta 2=0.02\end{array}$ \\
\hline Place of Residince & $\begin{array}{l}\text { With curfew ordi- } \\
\text { nances }\end{array}$ & $40.95 \pm 6.92$ & & $66.88 \pm 13.06$ & & $32.73 \pm 4.89$ & \\
\hline & $\begin{array}{l}\text { Without curfew } \\
\text { ordinances }\end{array}$ & $38.81 \pm 6.96$ & $\begin{array}{l}t=2.72 \\
p=0.007\end{array}$ & $68.86 \pm 11.74$ & $\begin{array}{l}\mathrm{t}=-3.54 \\
p=0.15\end{array}$ & $34.54 \pm 4.09$ & $\begin{array}{l}t=-1.43 \\
p<0.0001\end{array}$ \\
\hline Having Children & $\begin{array}{l}\text { Yes } \\
\text { No }\end{array}$ & $\begin{array}{l}40.22 \pm 6.94 \\
41.83 \pm 7.01\end{array}$ & $\begin{array}{l}t=-2.02 \\
p=0.04\end{array}$ & $\begin{array}{l}65.99 \pm 13.19 \\
73.78 \pm 8.80\end{array}$ & $\begin{array}{l}t=-7.25 \\
p<0.0001\end{array}$ & $\begin{array}{l}32.80 \pm 4.84 \\
34.69 \pm 4.17\end{array}$ & $\begin{array}{l}t=-3.37 \\
p=0.001\end{array}$ \\
\hline Income & Low $^{\mathrm{a}}$ & $42.26 \pm 6.71$ & $F=8.19$ & $63.56 \pm 14.99$ & $F=5.90$ & $32.09 \pm 5.09$ & $F=3.63$ \\
\hline Levels & $\begin{array}{l}\text { Moderate }^{b} \\
\text { High }^{c}\end{array}$ & $\begin{array}{l}40.73 \pm 7.02 \\
38.71 \pm 6.73\end{array}$ & $\begin{array}{l}p<0.0001 \\
a>c^{*}\end{array}$ & $\begin{array}{l}68.20 \pm 12.23 \\
68.45 \pm 11.57\end{array}$ & $\begin{array}{l}p=0.003 \\
b>a, \\
\quad c>a^{*} \prod^{2}=0.02\end{array}$ & $\begin{array}{l}33.57 \pm 4.93 \\
33.06 \pm 4.16\end{array}$ & $\begin{array}{l}p=0.02 \\
\eta^{2}=0.01\end{array}$ \\
\hline
\end{tabular}

PSS: Perceived Stress Scale; SSS: Spousal Support Scale; MLS: Marital Life Scale; M: Mean; S.D. Standart Deviation; F: One Way Analysis of Variance (ANOVA); $t$ : independent sample $t$-test; *Tukey test 
a higher mean SSS score than older participants $(F=7.91$, $p<0.0001)$. Male participants had a higher mean SSS score than female participants $(t=4.77, p<0.0001)$. Further, participants with a bachelor's degree had a higher mean SSS score than those without $(F=4.21, p=0.001)$. Participants without children had a higher mean SSS score than those with children $(t=-7.25, p<0.0001)$. Participants with high and moderate income had a higher mean SSS score than those with low income $(F=5.90, p=0.003)$. Age, sex, education level, place of residence, having children, and income affected participants' mean MLS score in Table 1 $(p<0.05)$. Younger participants had a higher mean MLS score than older participants $(F=6.36, p<0.0001)$. Male participants had a higher mean MLS score than female participants $(t=4.23, p<0.0001)$. Moreover, participants with a secondary school degree had a lower mean MLS score than others $(F=2.25, p=0.048)$. Participants living in cities with curfew ordinances had a higher mean MLS score than those who were not $(t=-3.54, p<0.0001)$. Participants who do not have children had a higher mean MLS score than those who have children $(t=-3.37, p=0.001)$. Moderate-income participants had a higher mean MLS score than those with low income $(F=3.63, p=0.02)$.
Age, sex, education level, and having children affected participants' mean CRSS subscale scores in Table 2. Female participants had a higher mean NCRS score than males $(t=-3.70, p<0.0001)$. Older participants had a higher mean SCRS score than younger participants $(F=6.13, p<0.0001)$. Male participants had a higher mean SCRS score than females $(t=4.08, p<0.0001)$. Participants with a primary school degree had a higher mean SCRS score than those with a higher degree $(F=5.44, p<0.0001)$. Participants with children had a higher mean Retreat Conflict Resolution Style (RCRS) score than those without $(t=2.15, p=0.03)$.

Table 3 presents the mean scale and subscale scores and the correlations between them. MLS was negatively correlated with PSS and NCRS and positively correlated with spousal support (emotional, instrumental, informational, appraisal, and social support) $(p<0.05)$. MLS was positively correlated with PCRS and SCRS $(p<0.05)$. PSS was negatively correlated with SSS and positively correlated with NCRS $(p<0.05)$. SSS was positively correlated with MLS and PCRS and negatively correlated with PSS and NCRS $(p<0.05)$.

Table 4 presents the correlation between the scale scores and anxiety levels during the COVID-19 pandemic.

Table 2 Conflict Resolution Styles Scale levels according to participants demographic characteristics

\begin{tabular}{|c|c|c|c|c|c|c|c|c|c|}
\hline $\begin{array}{l}\text { Demo- } \\
\text { graphic char- } \\
\text { acteristics }\end{array}$ & & $\begin{array}{l}\text { PCRS } \\
M \pm S . D\end{array}$ & $\begin{array}{l}\text { Analysis } \\
\text { Results }\end{array}$ & $\begin{array}{l}\text { NCRS } \\
M \pm S . D\end{array}$ & $\begin{array}{l}\text { Analysis } \\
\text { Results }\end{array}$ & $\begin{array}{l}\text { SCRS } \\
M \pm S . D\end{array}$ & $\begin{array}{l}\text { Analysis } \\
\text { Results }\end{array}$ & $\begin{array}{l}\text { RCRS } \\
M \pm S . D\end{array}$ & $\begin{array}{l}\text { Analysis } \\
\text { Results }\end{array}$ \\
\hline \multirow[t]{4}{*}{ Age } & $<30^{\mathrm{a}}$ & $28.07 \pm 4.95$ & $F=2.27$ & $16.83 \pm 6.50$ & $F=1.44$ & $23.63 \pm 5.48$ & $F=6.13$ & $23.43 \pm 7.45$ & $F=1.92$ \\
\hline & $30-40^{\mathrm{b}}$ & $26.97 \pm 5.05$ & $p=0.07$ & $17.82 \pm 6.61$ & $p=0.22$ & $21.89 \pm 6.71$ & $p<0.0001$ & $23.40 \pm 6.94$ & $p=0.12$ \\
\hline & $40-50^{c}$ & $26.34 \pm 4.82$ & $\eta^{2}=0.01$ & $17.05 \pm 5.46$ & $\eta^{2}=0.01$ & $23.92 \pm 6.29$ & $\mathrm{~d}>\mathrm{b}^{*}$ & $24.53 \pm 6.49$ & $\eta^{2}=0.01$ \\
\hline & $\geq 50^{\mathrm{d}}$ & $27.14 \pm 5.38$ & & $15.92 \pm 5.43$ & & $25.63 \pm 4.52$ & $\eta^{2}=0.04$ & $25.82 \pm 6.09$ & \\
\hline \multirow[t]{2}{*}{ Sex } & Male & $27.14 \pm 5.38$ & $t=0.48$ & $15,54 \pm 5,66$ & $t=-3.70$ & $24.93 \pm 6.22$ & $t=4.08$ & $22.89 \pm 7.09$ & $t=1.80$ \\
\hline & Female & $26.89 \pm 4.92$ & $p=0.63$ & $17.91 \pm 6.35$ & $p<0.0001$ & $22.29 \pm 6.30$ & $p<0.0001$ & $24.16 \pm 6.80$ & $p=0.07$ \\
\hline Education & Primary $^{\mathrm{a}}$ & $25.70 \pm 6.68$ & $F=1.82$ & $15.22 \pm 6.30$ & $F=1.55$ & $27.52 \pm 7.17$ & $F=5.44$ & $27.04 \pm 6.26$ & $F=2.60$ \\
\hline \multirow[t]{4}{*}{ Level } & Secondary ${ }^{\mathrm{b}}$ & $25.31 \pm 7.15$ & $p=0.10$ & $17.31 \pm 4.39$ & $p=0.17$ & $23.63 \pm 7.11$ & $p<0.0001$ & $23.50 \pm 6.80$ & $p=0.02$ \\
\hline & $\mathrm{High}^{\mathrm{c}}$ & $25.79 \pm 5.25$ & $\eta^{2}=0.02$ & $15.90 \pm 6.17$ & $\eta^{2}=0.02$ & $24.81 \pm 6.53$ & $a>b, a>c$ & $25.60 \pm 6.81$ & $a>b, a>c$ \\
\hline & Bachelor $^{\mathrm{d}}$ & $27.31 \pm 4.71$ & & $17.59 \pm 6.22$ & & $22.79 \pm 5.88$ & $a>d, a>e^{*}$ & $23.61 \pm 7.06$ & $a>e^{*}$ \\
\hline & $\begin{array}{l}\text { Post-Gradu- } \\
\text { ate }^{\mathrm{e}}\end{array}$ & $27.07 \pm 4.95$ & & $17.93 \pm 6.58$ & & $21.12 \pm 6.73$ & $\eta^{2}=0.05$ & $22.77 \pm 6.23$ & $\eta^{2}=0.03$ \\
\hline \multirow[t]{2}{*}{$\begin{array}{l}\text { Place of } \\
\text { Residince }\end{array}$} & $\begin{array}{r}\text { With curfew } \\
\text { ordinances }\end{array}$ & $26.78 \pm 4.99$ & $t=-1.52$ & $17.52 \pm 6.40$ & $t=1.44$ & $22.87 \pm 6.61$ & $t=-0.49$ & $23.85 \pm 7.03$ & $t=0.00$ \\
\hline & $\begin{array}{l}\text { Without } \\
\text { curfew } \\
\text { ordinances }\end{array}$ & $27.61 \pm 5.16$ & $p=0.12$ & $16.61 \pm 5.74$ & $p=0.17$ & $23.17 \pm 5.42$ & $p=0.66$ & $23.84 \pm 6.34$ & $p=0.99$ \\
\hline \multirow{2}{*}{$\begin{array}{l}\text { Having } \\
\text { Children }\end{array}$} & Yes & $26.80 \pm 5.03$ & $t=-1.57$ & $17.30 \pm 6.31$ & $t=-0.24$ & $23.03 \pm 6.36$ & $t=0.76$ & $24.14 \pm 6.93$ & $t=2.15$ \\
\hline & No & $27.73 \pm 5.01$ & $p=0.11$ & $17.48 \pm 6.09$ & $p=0.81$ & $22.45 \pm 6.47$ & $p=0.44$ & $22.40 \pm 6.50$ & $p=\mathbf{0 . 0 3}$ \\
\hline Income & Low & $27.31 \pm 5.70$ & $F=1.56$ & $16.91 \pm 6.30$ & $F=0.70$ & $23.70 \pm 6.40$ & $F=2.02$ & $24.20 \pm 7.22$ & $F=0.92$ \\
\hline \multirow[t]{2}{*}{ Levels } & Moderate & $27.15 \pm 4.55$ & $p=0.21$ & $17.23 \pm 6.03$ & $p=0.49$ & $23.07 \pm 6.23$ & $p=0.13$ & $24.07 \pm 6.67$ & $p=0.39$ \\
\hline & High & $26.33 \pm 5.31$ & $\eta^{2}=0.01$ & $17.81 \pm 6.68$ & $\eta^{2}=0.003$ & $22.12 \pm 6.56$ & $\eta^{2}=0.001$ & $23.19 \pm 7.02$ & $\eta^{2}=0.004$ \\
\hline
\end{tabular}

PCRS: Positive Conflict Resolution Style; NCRS: Negative Conflict Resolution Style; SCRS: Subordination Conflict Resolution Style; RCRS: Retreat Conflict Resolution Style M: Mean; S.D. Standart Deviation; F: One Way Analysis of Variance (ANOVA); $t$ : independent sample t-test; *Tukey test 


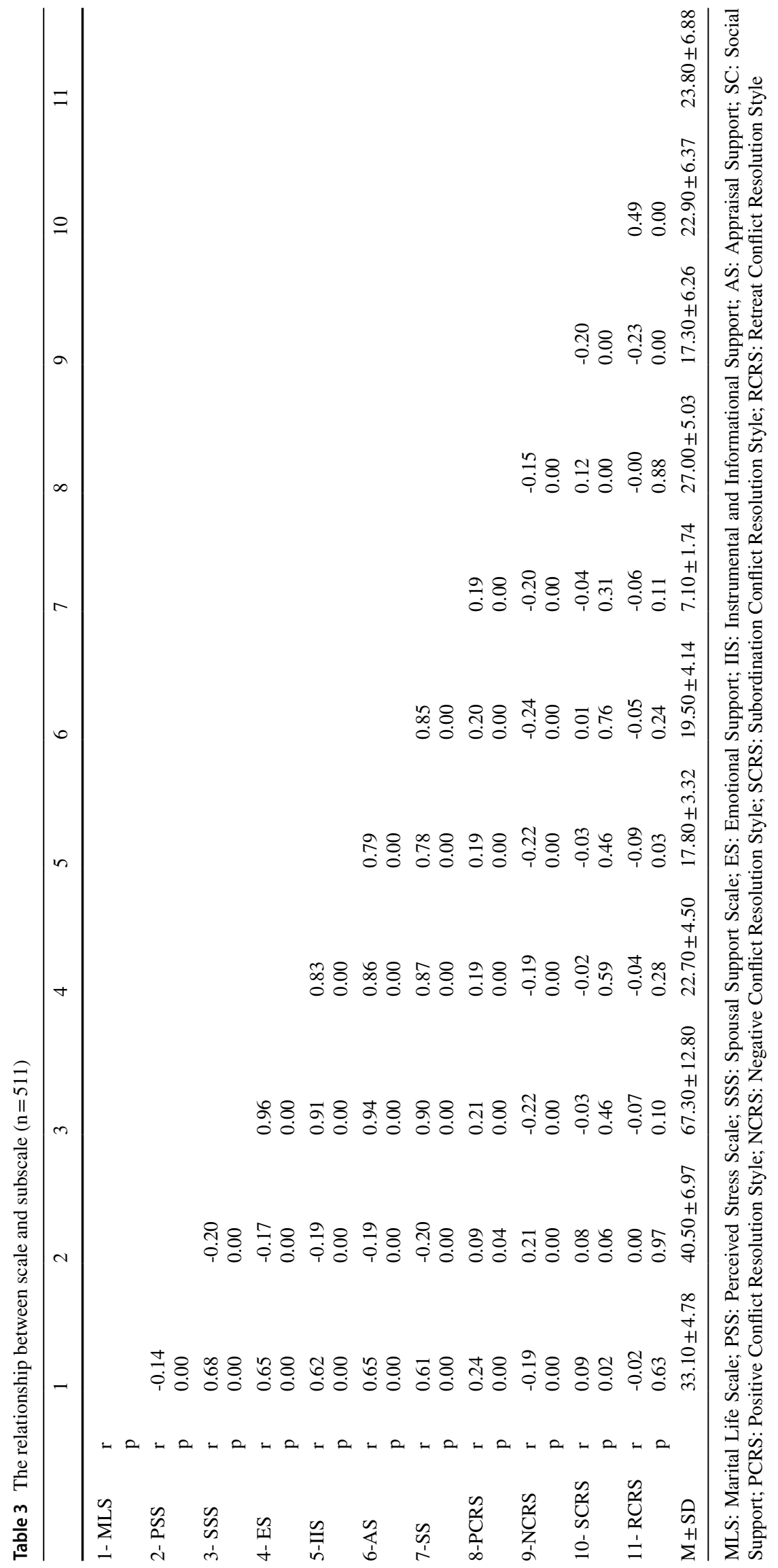


Table 4 The relationship between anxiety levels and scale scores

\begin{tabular}{|c|c|c|c|c|}
\hline & & $\begin{array}{l}\text { Anxiety Levels Onset of } \\
\text { the COVID-19 }\end{array}$ & $\begin{array}{l}\text { Anxiety Levels Last } \\
\text { Week }\end{array}$ & $\begin{array}{l}\text { Anxiety } \\
\text { Levels about } \\
\text { Health }\end{array}$ \\
\hline \multirow[t]{2}{*}{ PSS } & $\mathrm{r}$ & 0.24 & 0.21 & 0.31 \\
\hline & $\mathrm{p}$ & $0.00^{* *}$ & $0.00^{* *}$ & $0.00^{* *}$ \\
\hline \multirow[t]{2}{*}{ MLP } & $\mathrm{r}$ & -0.02 & 0.08 & 0.01 \\
\hline & $\mathrm{p}$ & 0.56 & 0.06 & 0.74 \\
\hline \multirow[t]{2}{*}{ SSS } & $\mathrm{r}$ & -0.06 & 0.044 & -0.00 \\
\hline & $\mathrm{p}$ & 0.16 & 0.32 & 0.93 \\
\hline \multirow[t]{2}{*}{ Emotional Support } & $\mathrm{r}$ & -0.06 & 0.05 & -0.00 \\
\hline & $\mathrm{p}$ & 0.13 & 0.23 & 0.94 \\
\hline \multirow{2}{*}{$\begin{array}{l}\text { Instrumental and Informa- } \\
\text { tional Support }\end{array}$} & $\mathrm{r}$ & -0.04 & 0.02 & 0.00 \\
\hline & $\mathrm{p}$ & 0.32 & 0.62 & 0.85 \\
\hline \multirow[t]{2}{*}{ Appraisal Support } & $\mathrm{r}$ & -0.04 & 0.06 & 0.00 \\
\hline & $\mathrm{p}$ & 0.36 & 0.15 & 0.84 \\
\hline \multirow[t]{2}{*}{ Social Support } & $\mathrm{r}$ & -0.09 & 0.02 & -0.05 \\
\hline & $\mathrm{p}$ & $0.02^{*}$ & 0.56 & 0.20 \\
\hline \multirow[t]{2}{*}{ PCRS } & $\mathrm{r}$ & 0.02 & 0.01 & 0.04 \\
\hline & $\mathrm{p}$ & 0.54 & 0.66 & 0.29 \\
\hline \multirow[t]{2}{*}{ NCRS } & $\mathrm{r}$ & 0,11 & -0.01 & 0.09 \\
\hline & $\mathrm{p}$ & $0.01^{*}$ & 0.71 & $0.03^{*}$ \\
\hline \multirow[t]{2}{*}{ SCRS } & $\mathrm{r}$ & -0.01 & 0.01 & 0.01 \\
\hline & $\mathrm{p}$ & 0.82 & 0.72 & 0.74 \\
\hline \multirow[t]{2}{*}{ RRCS } & $\mathrm{r}$ & -0.03 & -0.01 & 0.00 \\
\hline & $\mathrm{p}$ & 0.49 & 0.76 & 0.97 \\
\hline
\end{tabular}

MLS: Marital Life Scale; PSS: Perceived Stress Scale; SSS: Spousal Support Scale; PCRS: Positive Conflict Resolution Style; NCRS: Negative Conflict Resolution Style; SCRS: Subordination Conflict Resolution Style; RCRS: Retreat Conflict Resolution Style * $p<0,05 * * p<0,01$
Participants' anxiety at the onset of the COVID-19 pandemic was positively correlated with PSS and NCRS and negatively correlated with social support. Their anxiety in the last week was positively correlated with PSS, while their anxiety regarding their health was positively correlated with PSS and NCRS.

A multiple regression analysis was used to determine the effect of perceived stress, spousal support, and conflict resolution style on marital satisfaction. The results revealed that the model fit the data $(F=53.88 ; p<0.001)$ (Table 5). Positive and subordination conflict resolution styles and emotional, instrumental, and appraisal spousal support accounted for $48.30 \%$ of the total variance of marital satisfaction. Emotional spousal support had the largest effect on marital satisfaction (beta=0.25). A one-unit increase in the SSS "emotional" subscale was associated with an increase of 0.27 units in the MLS score.

\section{Discussion}

The COVID-19 pandemic is a global crisis that has caused physical, mental, social, behavioral, and economic problems worldwide. People have been suffering not only from health-related anxiety but also from stress, uncertainty, depression, and social isolation. These adverse effects have resulted in family conflicts and domestic violence on one hand but have also enhanced support and bonding between couples on the other. This study investigated the relationship among perceived stress, conflict resolution styles, spousal support, and marital satisfaction during the COVID-19 quarantine. It was found that the anxiety levels of married participants had increased due to the emotional, mental, social, and economic difficulties they had experienced as a result of the pandemic; this caused problems in their marital life. It was found that with the increased stress levels of spouses, spousal support and marital satisfaction decreased, and that as spousal support increased, marital satisfaction and positive conflict resolution increased. Further, the regression analysis revealed that positive conflict resolution and spousal support have significant associations with marital satisfaction. The most important finding was that the level of emotional support that spouses gave to each other in a situation of increased stress and anxiety had a more predictive effect on marital satisfaction than the other factors that were studied. The results of the study are discussed in line with the literature. 
Table 5 The effect of scale scores on marital life scales

\begin{tabular}{|c|c|c|c|c|c|c|c|}
\hline \multicolumn{8}{|l|}{ ANOVA } \\
\hline Model & \multicolumn{2}{|c|}{ Sum of Squares } & df & Mean Square & \multicolumn{2}{|l|}{$F$} & $p$ \\
\hline Regression & \multicolumn{2}{|c|}{5743.89} & 9 & 638.21 & \multicolumn{2}{|l|}{53.88} & $<0.0001$ \\
\hline Residual & \multicolumn{2}{|c|}{5934.28} & 501 & 11.84 & & & \\
\hline Total & \multicolumn{2}{|c|}{$11,678.18$} & 510 & & & & \\
\hline \multirow[t]{2}{*}{ Model } & \multicolumn{2}{|c|}{ Unstandardized Coefficients } & $\begin{array}{r}\text { Standardized } \\
\text { Coefficients }\end{array}$ & $t$ & $p$ & \multicolumn{2}{|c|}{ Confidence Interval (95.0\%) } \\
\hline & $\mathrm{B}$ & Standard Error & Beta & & & Lower Bound & Upper Bound \\
\hline Constant & 13.27 & 1.81 & & 7.32 & $<0.0001$ & 9.71 & 16.83 \\
\hline PSS & -0.00 & 0.02 & -0.01 & -0.34 & 0.73 & -0.05 & 0.03 \\
\hline PCRS & 0.08 & 0.03 & 0.08 & 2.54 & 0.01 & 0.01 & 0.14 \\
\hline NCRS & -0.00 & 0.02 & -0.01 & -0.28 & 0.77 & -0.06 & 0.04 \\
\hline SCRS & 0.09 & 0.02 & 0.12 & 3.26 & 0.00 & 0.03 & 0.14 \\
\hline RCRS & -0.02 & 0.02 & -0.04 & -1.09 & 0.27 & -0.08 & 0.02 \\
\hline Emotional Support & 0.27 & 0.08 & 0.25 & 3.18 & 0.00 & 0.10 & 0.44 \\
\hline $\begin{array}{l}\text { Instrumental and } \\
\text { Informational Sup- } \\
\text { port }\end{array}$ & 0.24 & 0.08 & 0.16 & 2.71 & 0.00 & 0.06 & 0.41 \\
\hline Appraisal Support & 0.27 & 0.08 & 0.24 & 3.39 & 0.00 & 0.11 & 0.44 \\
\hline Social Support & 0.10 & 0.19 & 0.03 & 0.52 & 0.60 & -0.28 & 0.49 \\
\hline
\end{tabular}

$\mathrm{R}=0.70 \mathrm{R}^{2}=0.492$ Adjusted. $\mathrm{R}^{2}=0.48 \mathrm{~F}=53.88$, Durbin Watson $=1.976, \mathrm{~N}=511, p<0.0001$

PSS: Perceived Stress Scale; PCRS: Positive Conflict Resolution Style; NCRS: Negative Conflict Resolution Style; SCRS: Subordination Conflict Resolution Style; RCRS: Retreat Conflict Resolution Style

We believe that the results of this study will help family experts develop and implement strategies to help couples in times of uncertainty and crisis (Stanley \& Markman, 2020). For participants in this study, the challenges of the COVID19 quarantine were emotional and mental stress, having to postpone important plans, financial loss or job loss, and marriage and family problems. The participants had anxiety scores above the normal range at the onset of the COVID-19 pandemic. Although their scores decreased upon the launch of controlled normalization in June 2020, they were nevertheless higher than the normal range. Social isolation during the COVID-19 pandemic has been challenging for people worldwide. People have been experiencing significantly higher levels of stress, anxiety, and depression during this period and also attempting to deal with deteriorating family relationships due to economic problems and employment concerns since the onset of the pandemic (James Riegler et al., 2020; Lebow, 2020; Wang et al., 2020).

Further, demographic characteristics were linked to participants' PSS, SSS, and MLS scores. Female participants had a higher mean PSS score, probably because they were more responsible for cooking, cleaning, and taking care of children, and ensuring that their education was not interrupted during the COVID-19 quarantine. Participants living in cities with curfew ordinances had a higher mean PSS score because the stay-at-home orders of 48-96 h contributed to more stress. Low-income participants had a higher mean PSS score as they faced financial problems due to layoffs and lockdowns. Altuntaş and Tekeci (2020) found that the COVID-19 pandemic contributed to more anxiety in males than in females. However, certain studies report the opposite (Duan \& Zhu, 2020; Wang et al., 2020; Zhang et al., 2020).

Younger participants had a higher mean SSS score probably because they were newlyweds. Male participants had a higher mean SSS score than females during the COVID-19 lockdown. In Turkish culture, females are typically responsible for household decisions; the added stress of the lockdown may be linked to lower SSS in females, as the lockdown would have complicated daily stress in the home (e.g., through things like having children at home during the day). Further, participants with a bachelor's degree had a higher mean SSS score, as education makes people more sensitive to gender equality and equal sharing of household tasks. Participants without children had a higher mean SSS score, probably because they were newlyweds and, therefore, more likely to support each other and empathize with each other. High-income participants had a higher mean SSS score, probably due to increased levels of awareness due to higher education. Günsel (2013) found that males and those with children had higher SSS scores, but that spousal support scores increased with age. 
Younger participants had a higher mean MLS score, probably because they were newlyweds with lower expectations and more conflict avoidance. Taşköprü (2013) also reported a negative correlation between the duration of marriage and marital satisfaction, but Çelik (2006) found no relationship between the two. Male participants had a higher mean MLS score, probably because they did not pull their weight in household chores during the COVID-19 lockdown. Further, a positive correlation was found between education level and marital satisfaction (Kaya, 2017) because people with higher education are likely to cope with stress better, thereby resulting in marital satisfaction (Çelik, 2006). Participants living in cities with curfew ordinances had a higher mean MLS score, probably because they were mostly big cities where couples adopted a more egalitarian approach to the division of household tasks. Participants without children had a higher mean MLS score, probably because they devoted their time and energy to their own needs, while those with children had to deal with their children's needs and problems, making them less satisfied with their marriage. Twenge et al. (2003) also reported that couples with children were less satisfied with their marriage than those without children. Moderate-income participants had a higher mean MLS score, probably because they faced fewer financial problems than people with low income.

Female participants had a higher mean NCRS score than males. Although we did not measure this, females may have been more likely to to respond to conflicts with verbal and physical aggression during the COVID-19 lockdown (Çakmak Tolan, 2015) because they felt the toll of juggling between work and family responsibilities, This is a conjecture which needs to be investigated further. Older participants had a higher mean SCRS score, probably because they were more likely to acquiesce to their partners' demands, particularly in times of stress, like social isolation. Male participants had a higher mean SCRS score, thereby suggesting that they might have made compromises instead of remaining headstrong in a discussion or an argument during the COVID-19 lockdown. This is merely a conjecture, and it requires additional study, as do many of our suggested conclusions. Participants with a primary school degree had a higher mean SCRS score probably because they turned to social values and traditions to resolve their marital conflicts. Older participants had a higher mean RCRS score, probably because they tended to respect their partners' opinions and make compromises instead of arguing. Participants with children had a higher mean RCRS score, probably because they avoided arguing in front of their children during the COVID-19 lockdown.

Further, marital satisfaction was negatively correlated with perceived stress and negative conflict resolution style and positively correlated with spousal support (emotional, instrumental, informational, appraisal, and social support).
On the other hand, marital satisfaction was positively correlated with positive and subordination conflict resolution styles. Spousal support was negatively correlated with perceived stress and negative conflict resolution style. Moreover, economic problems, instability, uncertainty, anxiety, fear, and scarce social support during the COVID-19 pandemic disrupted family life (Cluver et al., 2020; Usher et al., 2020). Pieh et al. (2020) also reported a negative correlation between perceived stress and marital satisfaction and spousal support during the COVID-19 lockdown.

Participants with higher levels of anxiety at the onset of the COVID-19 pandemic and in the last week of lockdown and those with higher levels of anxiety regarding health, in general, had higher PSS scores. In addition, those with higher anxiety levels at the onset of the COVID-19 pandemic and those generally anxious regarding health had higher NCRS scores. Those with higher anxiety levels at the onset of the COVID-19 pandemic had lower SSS "social support" subscale scores. People had also experienced increased stress levels during the outbreak of the severe acute respiratory syndrome (SARS) in 2002-2004 (Chua et al., 2004; Lee et al., 2007). Shevlin et al. (2020) found that the COVID-19 pandemic contributed to more stress in married couples with children. The COVID-19 pandemic and its repercussions in daily life caused uncertainty, stress, panic, and fear (Karataş, 2020), exacerbated by news reports regarding the pandemic, economic problems (Cluver et al., 2020), the fear of becoming infected and losing loved ones, prolonged physical and social isolation (Ahorsu et al., 2020; Brooks et al., 2020; de Lima et al., 2020), and rapid changes in daily life during the lockdowns and quarantine periods (Cluver et al., 2020). Those adverse experiences led to a rise in marital conflict, thereby resulting in couples denying each other the support they needed.

The multiple regression analysis revealed that marital satisfaction was significantly affected by positive conflict resolution styles and spousal support. In general, research shows a positive correlation between spousal support and marital satisfaction (Çağ \& Yıldırım, 2013; Kabasakal \& Soylu, 2016; Yedirir \& Hamata, 2015). A few studies also report that positive conflict resolution styles predict marital satisfaction (Çakmak Tolan, 2015; Erdem Özkan, 2019).

\section{Conclusion}

The COVID-19 pandemic has resulted in a global standstill, with massive political, economic, and social effects on countries and psychosocial effects on individuals. Couples have found themselves navigating new problems brought about by the pandemic and the preventive measures taken by countries to curb its spread. Therefore, experts must enable couples 
to develop strategies to cope with stressors and resolve conflicts in times of crisis; couples must be encouraged to support each other and build a strong bond. Such strategies will help families and, thus, the entire society overcome such unforeseen crises. It is recommended that future studies be conducted with larger sample and different cultural groups, as well as qualitative and mixed methods research to evaluate the experiences of the spouses more deeply. At the same time, the impact of the pandemic process on spouses should be evaluated by longitudinal studies. Future studies can also add more and new variables.

\section{Limitation of the Study}

This study had three limitations. First, it was a web-based study that employed the snowball sampling method. Therefore, the sample consisted of people from similar socioeconomic status who could complete online surveys. Second, the sample was demographically homogeneous and, therefore, the results cannot be generalized to the entire population. Third, this was a correlational and descriptive cross-sectional study. Longitudinal investigations must be conducted in future studies to assess the effects of the COVID-19 pandemic. Future studies could also employ qualitative research methods and recruit people representing all socioeconomic groups to assess the predictors of marital satisfaction during the COVID-19 pandemic.

Data availability The datasets generated during and/or analyzed during the current study are available from the corresponding author on reasonable request.

\section{Declarations}

Ethical Approval All procedures performed in studies involving human participants were in accordance with the ethical standards of the institutional and/or national research committee and with the 1964 Helsinki declaration and its later amendments or comparable ethical standards.

Informed Consent Informed consent was obtained from all individual participants included in the study.

Conflict of Interest The authors personally have not received any funding for this project. Therefore, the authors declare that no conflict of interest exists.

\section{References}

Abbas, J., Aqeel, M., Abbas, J., Shaher, B., Jaffar, A., Sundas, J., \& Zhang, W. (2019). The moderating role of social support for marital adjustment, depression, anxiety, and stress: Evidence from Pakistani working and nonworking women. Journal of Affective
Disorders, 244, 231-238. https://doi.org/10.1016/j.jad.2018.07. 071

Ahorsu, D. K., Lin, C. Y., Imani, V., Saffari, M., Griffiths, M. D., \& Pakpour, A. H. (2020). The fear of COVID-19 scale: Development and initial validation. International Journal of Mental Health and Addiction. https://doi.org/10.1007/s11469-020-00270-8

Akbaş, Ö. Z., \& Dursun, C. (2020). Koronavirüs (COVID-19) pandemisi sürecinde özel alanına kamusal alanı sı̆̆dıran çalışan anneler. Avrasya Sosyal Ve Ekonomi Araştırmaları Dergisi, 7(5), 78-94.

Altuntaş, O., \& Tekeci, Y. (2020). Effect of COVID 19 on perceived stress, coping skills, self-control and self-management skills. Research Square. https://doi.org/10.21203/rs.3.rs-48393/v1

Armstrong, A. J., Holmes, C. M., \& Henning, D. (2020). A changing world, again. How appreciative inquiry can guide our growth. Social Sciences \& Humanities Open, 2(1). https://doi. org/10.1016/j.ssaho.2020.100038

Brooks, S. K., Webster, R. K., Smith, L. E., Woodland, L., Wessely, S., Greenberg, N., \& Rubin, G. J. (2020). The psychological impact of quarantine and how to reduce it: Rapid review of the evidence. The Lancet, 395(10227), 912-920. https://doi.org/10.1016/S01406736(20)30460-8

Centers for Disease Control and Prevention (CDC). (2020). Coronavirus disease 2019 (COVID-19) situation summary. https://www. cdc.gov/coronavirus/2019-ncov/index.html. Accessed 9 Oct 2020.

Chua, S. E., Cheung, V., McAlonan, G. M., Cheung, C., Wong, J. W., Cheung, E. P., Chan, M. T., Wong, T. K., Choy, K. M., Chu, C. M., Lee, P. W., \& Tsang, K. W. (2004). Stress and psychological impact on SARS patients during the outbreak. The Canadian Journal of Psychiatry, 49(6), 385-390. https://doi.org/10.1177/ 070674370404900607

Cluver, L., Lachman, J. M., Sherr, L., Wessels, I., Krug, E., Rakotomalala, S., Blight, S., Hillis, S., Bachman, G., Green, O., Butchart, A., Tomlinson, A., Ward, C. L., Doubt, J., \& McDonald, K. (2020). Parenting in a time of COVID-19. The Lancet, 395(10231). https://doi.org/10.1016/S0140-6736(20)30736-4.

Cohen, S., Kamarck, T., \& Mermelstein, R. (1983). A global measure of perceived stress. Journal of Health and Social Behavior, 24, 385-396. https://doi.org/10.2307/2136404

Çă̆, P., \& Yıldırım, İ. (2013). Evlilik doyumunu yordayan ilişkisel ve kişisel değişkenler. Türk Psikolojik Danışma Ve Rehberlik Dergisi, 4(39), 13-23.

Çakmak Tolan, Ö. (2015). Evlilik uyumunun kişilik özellikleri, ilişkiye dair inançlar ve çatışma çözüm stilleri bağlamında yordanması (Diyarbakır ili örne ği) [Unpublished doctoral dissertation, İnönü Üniversitesi]. https://tez.yok.gov.tr/UlusalTezMerkezi/. Accessed 12 Oct 2020.

Çelik, M. (2006). Evlilk Doyumu Ölçeğini geliștirme çalışması [Unpublished doctoral dissertation, Çukurova Üniversitesi]. https://tez. yok.gov.tr/UlusalTezMerkezi/. Accessed 12 Oct 2020.

de Lima, C. A., Alves, P. M. R., de Oliveira, C. J. B., de Oliveira, T. R. N., Barbosa, K. B., Marcene, H. C., \& de Oliveira, S. V. (2020). Letter to the editor: COVID-19: Isolations, quarantines and domestic violence in rural areas. SciMedicine Journal, 2(1), 44-45. https://doi.org/10.28991/SciMedJ-2020-0201-7

Duan, L., \& Zhu, G. (2020). Psychological interventions for people affected by the COVID-19 epidemic. The Lancet, 7(4), 300-302. https://doi.org/10.1016/S2215-0366(20)30073-0

Erdem-Özkan, G. (2019). Evli bireylerin çatışma çözüm stillerine göre evlilik doyumunun incelenmesi [Unpublished master's thesis, Ege Üniversitesi]. https://tez.yok.gov.tr/UlusalTezMerkezi/. Accessed 12 Oct 2020.

Eskin, M., Harlak, H., Demirkıran, F., \& Dereboy, Ç. (2013). Algılanan Stres Ölçeğinin Türkçeye uyarlanması: Güvenirlik ve geçerlik analizi. New Symposium Journal, 51(3), 132-140.

Gulati, G., \& Kelly, B. D. (2020). Domestic violence against women and the COVID-19 pandemic: What is the role of psychiatry? 
International Journal of Law and Psychiatry. https://doi.org/10. 1016/j.ijlp.2020.101594

Günsel, D. A. (2013). Evli bireylerin algıladıkları eş desteğinin çeşitli değişkenler açısından değerlendirilmesi: KKTC örne ği [Unpublished doctoral dissertation, Ankara Üniversitesi]. https://tez.yok. gov.tr/UlusalTezMerkezi/. Accessed 12 Oct 2020.

Huang, Y., \& Zhao, N. (2020). Generalized anxiety disorder, depressive symptoms and sleep quality during COVID-19 outbreak in China: A web-based cross-sectional survey. Psychiatry Research. https:// doi.org/10.1016/j.psychres.2020.112954

James Riegler, L., Raj, S. P., Moscato, E. L., Narad, M. E., Kincaid, A., \& Wade, S. L. (2020). Pilot trial of a telepsychotherapy parenting skills intervention for veteran families: Implications for managing parenting stress during COVID-19. Journal of Psychotherapy Integration, 30(2), 290-303. https://doi.org/10.1037/int0000220

Kabasakal, Z., \& Soylu, Y. (2016). Evli bireylerin evlilik doyumunun cinsiyet ve eş desteğine göre incelenmesi. Ĕ̆itim Ve Ö̆gretim Araștırmaları Dergisi, 5(4), 208-214.

Karataş, Z. (2020). COVID-19 Pandemisinin toplumsal etkileri, değişim ve güçlenme. Türkiye Sosyal Hizmet Araştırmaları Dergisi, 4(1), 3-17.

Kaya, N. (2017). Kırsal ve kentsel alanda yaşayan evli kadınların evlilik иуuтu, evlilik doyumu ve ilişkilerde mutluluk düzeylerinin karşılaştırılması ve incelenmesi [Unpublished master's thesis, İstanbul Ticaret Üniversitesi]. https://tez.yok.gov.tr/UlusalTezM erkezi/

Lebow, J. L. (2020). Family in the age of COVID-19. Family Process, 59(2), 309-312. https://doi.org/10.1111/famp.12543

Lee, A. M., Wong, J. G., McAlonan, G. M., Cheung, V., Cheung, C., Sham, P. C., Chu, C. M., Wong, P. C., Tsang, K. W. T., \& Chua, S. E. (2007). Stress and psychological distress among SARS survivors 1 year after the outbreak. The Canadian Journal of Psychiatry, 52(4), 233-240. https://doi.org/10.1177/070674370705200405

Liu, N., Zhang, F., Wei, C., Jia, Y., Shang, Z., Sun, L., Wu, L., Sun, Z., Zhou, Y., Wang, Y., \& Liu, W. (2020). Prevalence and predictors of PTSS during COVID-19 outbreak in China hardest-hit areas: Gender differences matter. Psychiatry Research, 287, 112921. https://doi. org/10.1016/j.psychres.2020.112921

Özen, A. (2006). Value similarities of wives and husbands and conflict resolution styles of spouses as predictors of marital adjustment. [Unpublished master thesis, Middle East Technical University].https://tez.yok.gov.tr/UlusalTezMerkezi/. Accessed 12 Oct 2020

Pieh, C., O’ Rourke, T., Budimir, S., \& Probst, T. (2020). Relationship quality and mental health during COVID-19 lockdown. Plos one, 15(9). https://doi.org/10.1371/journal.pone.023890.

Prime, H., Wade, M., \& Browne, D. T. (2020). Risk and resilience in family well-being during the COVID-19 pandemic. American Psychologist, 75(5), 631-643. https://doi.org/10.1037/amp0000660

Shakespeare-Finch, J., Bowen-Salter, H., Cashin, M., Badawi, A., Wells, R., Rosenbaum, S., \& Steel, Z. (2020). COVID-19: An Australian perspective. Journal of Loss and Trauma, 25(8), 662-672. https:// doi.org/10.1080/15325024.2020.1780748

Shevlin, M., McBride, O., Murphy, J., Miller, J.G., Hartman, T.K., Levita, L., Mason, L., Martinez, A. P., McKay, R., Stocks, T. V. A., Bennett, K. M., Hyland, P., Karatzias, T., \& Bentall, R. P. (2020). Anxiety, depression, traumatic stress, and COVID-19 related anxiety in the UK general population during the COVID-19 pandemic. PsyArXiv Preprints, 6(6). https://doi.org/10.31234/osf.io/hb6nq.

Soylu, Y., \& Kağnıcı, D. Y. (2015). Evlilik uyumunun empatik eğilim, iletişim ve çatışma çözme stillerine göre yordanması. Turkish Psychological Counseling \& Guidance Journal, 5(43), 44-54.

Stanley, S. M., \& Markman, H. J. (2020). Helping couples in the shadow of COVID-19. Family Process, 59(3), 937-955. https://doi.org/10. 1111/famp. 12575
Taşköprü, M. (2013). Evlilik doyumu ile problem çözme becerileri, stresle başa çıkma ve evlilik süresi arasındaki ilişkinin incelenmesi [Unpublished master's thesis, İstanbul Bilim Üniversitesi]. https://tez.yok. gov.tr/UlusalTezMerkezi/. Accesed 12 Oct 2020.

Tezer, E. (1994). Evli eşler arasındaki çatışmalar ile çeşitli demografik değişkenlerin evlilik doyumuna etkisi: Kadın eşin bir işte çalıştığ ve çalışmadığı eşler üzerinde bir araştırma. Psikiyatri, Psikoloji Ve Psikofarmakoloji Dergisi, 2(3), 209-217.

Twenge, J. M., Campbell, W. K., \& Foster, C. A. (2003). Parenthood and marital satisfaction: A meta-analytic review. Journal of Marriage and Family, 65(3), 574-583. https://doi.org/10.1111/j.1741-3737. 2003.00574.x

Usher, K., Bhullar, N., Durkin, J., Gyamfi, N., \& Jackson, D. (2020). Family violence and COVID- 19: Increased vulnerability and reduced options for support. International Journal of Mental Health Nursing. https://doi.org/10.1111/inm.12735

Ünal, B., \& Gülseren, L. (2020). COVID-19 pandemisinin görünmeyen yüzü: Aile içi kadına yönelik şiddet. Klinik Psikiyatri Dergisi, 23, 89-94. https://doi.org/10.5505/kpd.2020.37973

Wang, C., Pan, R., Wan, X., Tan, Y., Xu, L., Ho, C. S., \& Ho, R. C. (2020). Immediate psychological responses and associated factors during the initial stage of the 2019 coronavirus disease (COVID-19) epidemic among the general population in China. Environmental Research and Public Health, 17(5), 1729. https://doi.org/10.3390/ ijerph17051729

World Health Organization (WHO). (2020). Novel coronavirus (2019$\mathrm{nCoV}$ ): Situation report. Retrieved from https://www.who.int/docs/ default-source/coronaviruse/situationreports. Accessed 10 Oct 2020.

Wu, Z., \& McGoogan, J. M. (2020). Characteristics of and important lessons from the coronavirus disease 2019 (COVID-19) outbreak in China: Summary of a report of 72314 cases from the Chinese Center for Disease Control and Prevention. JAMA, 323(13), 1239-1242. https://doi.org/10.1001/jama.2020.2648

Xiang, Y. T., Yang, Y., Li, W., Zhang, L., Zhang, Q., Cheung, T., \& Ng, C. H. (2020). Timely mental health care for the 2019 novel coronavirus outbreak is urgently needed. The Lancet Psychiatry, 7(3), 228-229. https://doi.org/10.1016/S2215-0366(20)30046-8

Yıldırım, İ. (2004). Eş Destek Ölçeğinin geliştirilmesi. Türk Psikolojik Danışma ve Rehberlik Dergisi, 3(22), 19-26. https://doi.org/10. 17066/pdrd.95605

Yedirir, S., \& Hamarta, E. (2015). Emotional expression and spousal support as predictors of marital satisfaction: The case of Turkey. Educational Sciences: Theory \& Practice, 15(6), 1549-1558. https://doi. org/10.12738/estp.2015.6.2822

Zhang, W., Wang, K., Yin, L., Zhao, W., Xue, Q., Peng, M., Min, B., Tian, Q., Leng, H., Du, J., Chang, H., Yang, Y., Li, W., Shangguan, F., Yan, T., Dong, H., Han, Y., Wang, Y., Cosci, F., \& Wang, H. (2020). Mental health and psychosocial problems of medical health workers during the COVID-19 epidemic in China. Psychotherapy and Psychosomatics, 89(4), 242-250. https://doi.org/10.1159/00050 7639

Zhu, Z., Liu, Q., Jiang, X., Manandhar, U., Luo, Z., Zheng, X., Li, Y., Xie, J., \& Zhang, B. (2020). The psychological status of people affected by the COVID-19 outbreak in China. Journal of Psychiatric Research., 129, 1-7. https://doi.org/10.1016/j.jpsychires. 2020.05.026

Publisher's Note Springer Nature remains neutral with regard to jurisdictional claims in published maps and institutional affiliations. 